\title{
First Tests of MICROMEGAS and GEM-Like Detectors Made of a Resistive Mesh
}

\author{
R. Oliveira, V. Peskov, F. Pietropaolo, and P. Picchi
}

\begin{abstract}
We describe here various detector designs: GEM-type, MICROMEGAS-type, as well as cascaded detectors made of a resistive mesh manufactured from a resistive Kapton foil, $\left(20 \mu \mathrm{m}\right.$ thick, surface resistivity a few $\left.\mathrm{M} \Omega / \mathrm{cm}^{2}\right)$ by a laser drilling technique. As in any other micro-pattern detector, the maximum achievable gas gain of these detectors is restricted by the Raether limit; however, the resistive mesh makes them and the front end electronics fully spark protected. This approach may function as an alternative/or complementary component of the ongoing efforts in developing MICROMEGAS and GEMs with resistive anode readout plates and can be especially beneficial in micro-pattern detectors combined with a micro-pixel-type integrated front end electronics.
\end{abstract}

Index Terms-CsI photocathode, GEM, MICROMEGAS, micro-pattern detectors, resistive mesh.

\section{INTRODUCTION}

D UE to their very high granularity, recently developed micro-pattern gaseous detectors offer new possibilities in experimental techniques and applications and for this reason these detectors have generated great interest. However, the fine structures of their electrodes make the micro-pattern detectors very fragile. For example, damage by occasional sparks may easily materialize. Studies show (see [1], [2] and references therein) that in high quality detectors (no defects like dirty spots or sharp edges) sparks appear when the total charge in avalanche exceeds some critical value $Q_{\text {crit }}$ :

$$
\mathrm{A}_{\max } \mathrm{n}_{0}=\mathrm{Q}_{\text {crit }}
$$

where $A_{\max }$ is the maximum achievable gain before the breakdown occurs and $n_{0}$ is the number of primary electrons produced in the detector volume by the ionizing radiation. Note that a similar limit was empirically established quite a long time ago by $\mathrm{H}$. Raether [3] for large gap $(>3 \mathrm{~mm}$ ) parallel-plate avalanche chambers and usually is called the "Raether limit". However, it was recently shown [2] that a modified Raether

Manuscript received April 02, 2010; revised June 16, 2010 and August 04, 2010; accepted August 23, 2010. Date of current version December 15, 2010.

R. Oliveira is with the TS Division, CERN, CH-1211 Geneva-23, Switzerland (e-mail: rui.de.oliveira@cern.ch).

V. Peskov is with the V. Peskov is with the Institute for Nuclear Research, UNAM, Mexico City, MX-04510, Mexico, and also with the PH Division, CERN, CH-1211 Geneva-23 (e-mail: vladimir.peskov@ cern.ch).

F. Pietropaolo is with the INFN Padova, Padova, Italy, I-135131 (e-mail: francesco.pietropaolo@cern.ch).

P. Picchi is with INFN Frascati, Frascati, Italy I-10025 (e-mail: pio.picchi@cern.ch).

Digital Object Identifier 10.1109/TNS.2010.2073483 limit applies for every micropattern detectors: GEMs, MICROMEGAS and others. The physics behind this supports that at this critical value of $Q_{\text {crit }}$ the space charge in the avalanche becomes sufficient to disturb the external electric filed. As a result, photoelectrons created by avalanches in the surrounding gas volume begin to drift towards the region of positive ions remaining from the initial avalanche and finally form a thin plasma filament, called a streamer. The streamer touching the cathode creates a spark. Typically, for micro-pattern detectors depending on their design and operational pressure

$$
\mathrm{Q}_{\text {crit }}=10^{6}-10^{7} \text { electrons }
$$

It should be stressed that in practical situations to avoid an excessive number of potentially damaging sparks, one has to work at gas gains of one or two orders of magnitude smaller than the gains that correspond to the $A_{\max }$ estimated from the formula (1).Thus, if micro-pattern detectors are used to only detect radiation producing relatively small amounts of primary electrons $\mathrm{n}_{0} \sim 100$ (minimum ionizing particles, soft X-rays) they can reliably operate at gains $\leq 10^{3}$. However, in the presence of heavily ionizing particles and/or neutron background producing dense ionization with a very high value of $n_{0}$, the maximum, achievable gain will correspondingly drop.

In fact, the main problem appears during the long term operation of micro-pattern detectors: even if they operate at relatively low gains at some point a particle producing high values of $\mathrm{n}_{0}$ may appear (for example, due to the natural radioactivity), and this may trigger the breakdown. Moreover, at high counting rates there is also some probability of avalanches overlapping in time and space, which statistically creates $\mathrm{Q}_{\text {crit }}$ (and thus sparks) even at low gains. In addition to this effect, sporadic emissions of electron jets from the cathodes may result in another source of breakdowns (see [1]). As a result during the long-term operation of micro-pattern detectors, even at gas gains of $\mathrm{A} \ll \mathrm{A}_{\max }$, occasional sparks are almost unavoidable. It is obvious that in bad quality detectors or one damaged by discharges the sparks can be triggered by defects at gains/voltages below than that followed by formula (1). In light of these theoretical and experimental facts, one can expect that in real experimental conditions, as such a long term high energy physics experiment, GEMs and MICROMEGAS will always have some probability of sparking. The problem of discharges becomes much more severe in the case of photosensitive micropattern detectors oriented on such applications as RICH or dark-matter cryogenic detectors (see for example [4] and references therein) or special UV imaging deices [5]: they have to operate at gains of $10^{5}$ or more so as to detect single photoelectrons and thus any radioactive background creating $n_{0}>10$ electrons per 
event will cause breakdowns. Sustained efforts are focused on reducing this probability as much as possible and protecting detectors from damages by sparks. These measures include: segmentation of electrodes into several isolating parts (to reduce the active capacity involved in the discharge process), utilization of cascaded detectors (besides the lower voltages on each detector this also increases $Q_{\text {crit }}$ in the last detector stage due to the lateral diffusion of the electron cloud exiting the holes of the first and second stages of the cascaded detectors) [6]) and when possible, employment of spark-protected front end electronics. Unfortunately at this point, these efforts have produced limited success. For example in cascaded detectors, discharge propagations from on element to another one and to the readout plate have occurred; this effect has a higher probability of occurring in the presence of heavily ionizing particles [7]. Note that in the case of detectors combined with a multipixel SMOS array or MediPix (see [8], [9]) the requirements for the spark protection are exceptionally high due to the extreme fragility of this readout system.

Recently we proposed another, more radical solution for spark protection of micro-pattern detectors: the use of resistive electrodes instead of traditional metallic ones [10]. This approach was successfully implemented in the case of GEM-like detectors having a thickness $>0.2 \mathrm{~mm}$. Unfortunately, due to the mechanical instability of thin glass fiber sheets it was difficult to produce thinner GEMs-like detectors with resistive electrodes by the $\mathrm{CNC}$ drilling technique used to create a matrix of holes. We named this detector a Resistive Electrode Thick GEM or RETGEM. Resistive electrodes reduce the energy released by the sparks by 100-1000 times [11] and thus make these detectors as well as the front-end electronics spark-protected. Recently, several groups working with such detectors fully confirmed our earlier results [12]-[14]. Unfortunately, the position resolution of RETGEM (similarly to TGEM) is limited by the hole's diameter and pitch and is about $0.7-1 \mathrm{~mm}$ [15], [16].

As a result of promising RETGEM experimentation, some other groups have begun investigating the possibility of applying the resistive electrode approach to MICROMEGAS; prototypes of detectors with anodes coated with various resistive layers were already developed and tested [17], [18]. Although these studies are still in progress, the preliminary results clearly indicate that spark protection can be achieved by this technique.

In this report we will present an alternative/complementary approach: detectors made of thin resistive meshes. From such resistive meshes one can assemble various detectors: GEM-like with gaps below $0.2 \mathrm{~mm}$, MICROMEGAS-like, as well as multistep detectors operating in cascaded mode. In addition to sparking protection these detectors offer a better position resolution than the current RETGEM designs. Preliminary results were reported in [19].

\section{MAterial AND MethodS}

\section{A. Resistive Mesh and Detector Designs}

We used resistive meshes (see Fig. 1) manufactured by a laser drilling technique from the resistive Kapton (surface resistivity

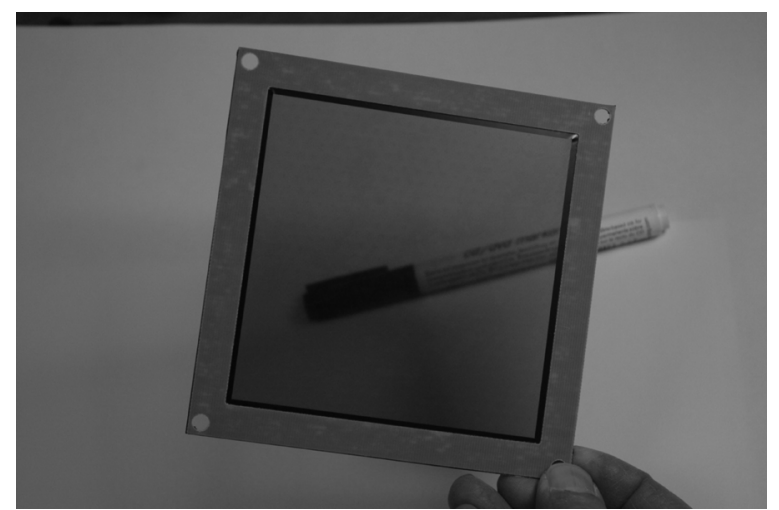

Fig. 1. A photograph of a resistive mesh manufactured by the laser drilling technique.

2.8-3.0 M $\Omega / \mathrm{cm}^{2}$ ) used earlier by us in the RETGEM designs [10]. The resistive meshes had a thickness of $20 \mu \mathrm{m}$, holes' diameter of $50 \mu \mathrm{m}$ and hole spacing of $100 \mu \mathrm{m}$.

The resistive meshes were stretched either on $5 \times 5 \mathrm{~cm}^{2}$ or $10 \times 10 \mathrm{~cm}^{2} 0.4 \mathrm{~mm}$ thick rectangular fiber glass frames. From these stretched meshes different detectors were assembled: MICROMEGAS-like (Fig. 2(a)), GEMs-like (2(b)) or cascaded detectors (see Fig. 3). Here we named these detectors "MIROMEGAS-like" and "GEM-like" because geometrically they are resemble the respective detectors; obviously they may have (especially "GEM"-like) different field line maps than classical MICROMEGAS or GEMs. Two types MICROMEGAS-like designs were tested (see Fig. 2(a)): a "home-made" one, assembled by us in the laboratory, and so-called "bulk" one manufactured in the DEM-PMT Workshop at CERN by the technology described in [20]. In the home-made detectors we could easily change the geometry, spacers and cetera and for this reason they were mainly used for general studies and for the detector designs optimization. We have two main design of the "home-made" detectors: thin-gap (gaps, or be more precise, the spacer width were $\mathrm{G}=50 \mu \mathrm{m}$ or $100 \mu \mathrm{m})$ and the thick-gap detectors $(\mathrm{G}=200 \mu \mathrm{m}, 300 \mu \mathrm{m}$ or $500 \mu \mathrm{m}$.) The thin-gap detector maintains the same design as the conventional one with the exception that its cathode was made from the resistive mesh described above. As anodes we used either metallic plates or fiber glass plates covered with resistive Kapton or (for position resolution measurements) a ceramic plate with a $50 \mu \mathrm{m}$ pitch metallic strips described in [21]. As spacers between the meshes and the anode plate we used $50-300 \mu \mathrm{m}$ thick Nylon fishing lines, or $50 \mu \mathrm{m}$ thick meshes made of standard Kapton by a photolithographic technique (cell sizes 1 or $2 \mathrm{~mm}$ ).

In the case of the most of the thick-gap designs we used spacer only outside the active area, for example in the region of the fiber glass frames (we of course tested some designs with spacers inside the active area, see Section III).

Bulk MICROMEGAS-like detector with resistive cathode mesh has an active area of $5 \times 5 \mathrm{~cm}^{2}$ and a gap $\mathrm{G}=0.13 \mathrm{~mm}$. It was built to demonstrate that a detector with incorporated supporting pillars can be manufactured which opens the possibility to build large-area high quality detectors by industrial process (see [22] for more details). 


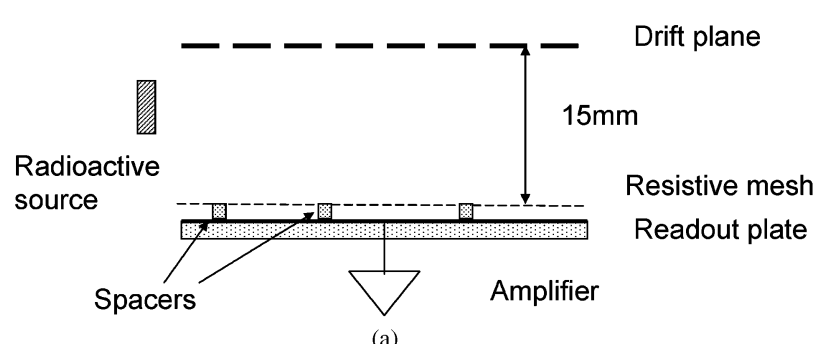

(a)

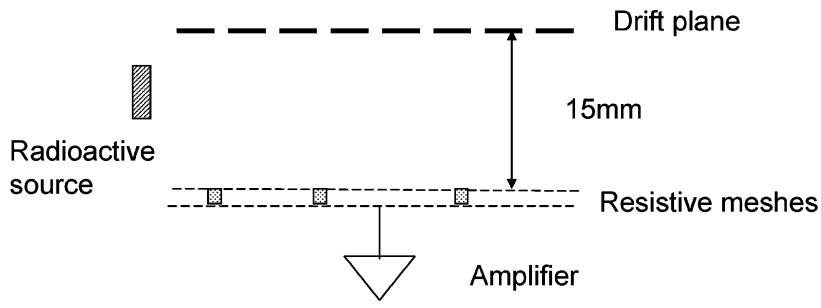

(b)

Fig. 2. A schematic drawing of MICROMEGAS-like detector with resistive cathode mesh (a) and GEM-like detector assembled from two resistive meshes (b).

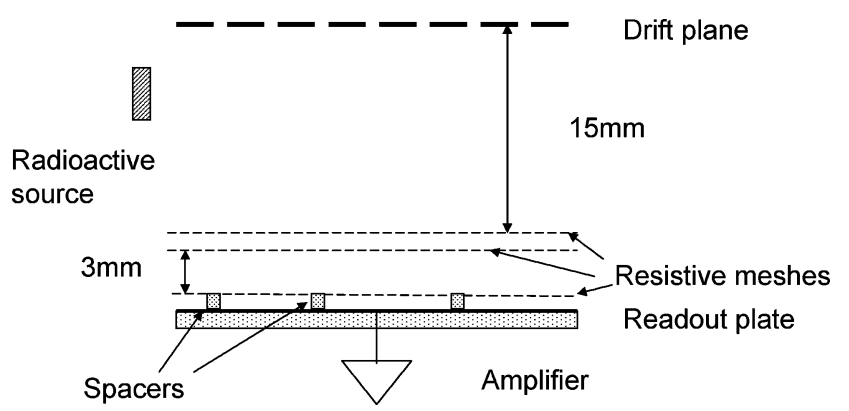

(a)

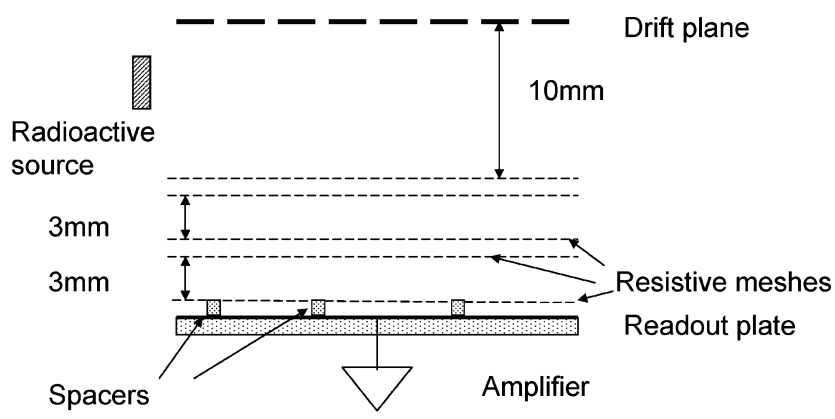

(b)

Fig. 3. Schematic drawing of a cascaded detector based on resistive mesh: (a) RM-MICROMEGAS combined with RM-GEM preamplification structure, (b) RM-MICROMEGAS combined with two cascaded RM-GEMs.

Henceforth we will call detectors with resistive cathode meshes described above a Resistive Mesh MICROMEGAS or $\mathrm{RM}-\mu \mathrm{M}$.

Resistive mesh GEMs-like detectors (RM-GEMs) were manufactured from two parallel resistive meshes: one served as the anode and the other one as the cathode. As in the case of RM-MICROMEGAS the thin gap RM-GEMs $(\mathrm{G}=50$ or $100 \mu \mathrm{m})$ fishing line spacers were placed between the meshes

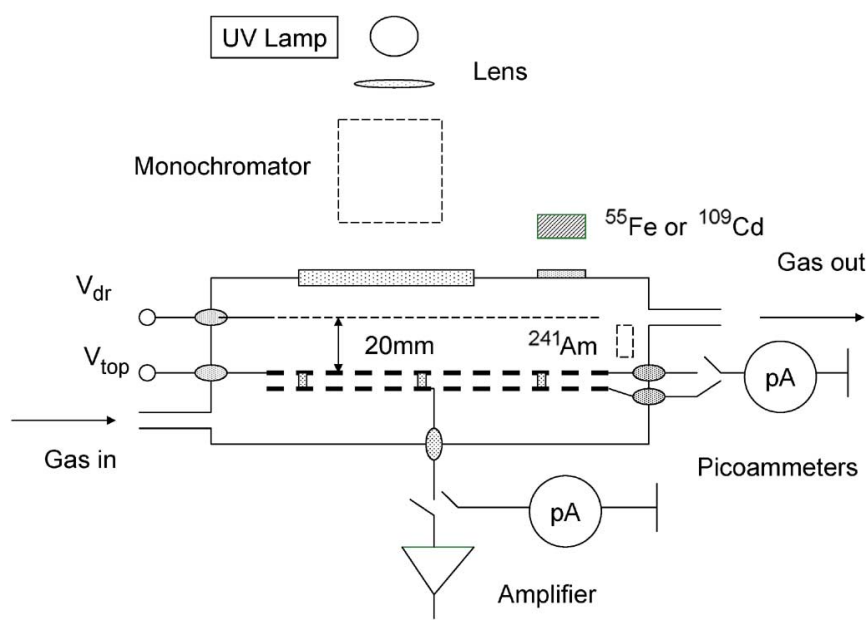

Fig. 4. A schematic drawing of the experimental setup.

whereas in the case of a thick gap RM-GEMs $(\mathrm{G}=200 \mathrm{~m})$ both the fishing lines and the Kapton frame were used based upon particular design.

In some tests one of the resistive meshes was coated with an $0.4 \mu \mathrm{m}$ thick CsI layer.

The cascaded detector consisted from an $\mathrm{RM}-\mu \mathrm{M}$ $(\mathrm{G}=0.1 \mathrm{~mm})$ combined with one or two RM-GEM preamplifiers $(\mathrm{G}=0.2 \mathrm{~mm})$. The distance between the RM-GEMs and the $\mathrm{RM}-\mu \mathrm{M}$ was $3-4 \mathrm{~mm}$.

\section{B. Experimental Setup}

The experimental setup consisted of a gas chamber housing one of the above mentioned detectors, an $\mathrm{Ar}(\mathrm{Hg}$ ) UV lamp (for position resolution measurements a pulsed $\mathrm{D}_{2}$ lamp was also used), a monochromator, a lens focusing the light from the lamp to the input slit of the monochromator (or directly to the top surface of RM- $\mu \mathrm{M}$ in the case of position-resolution measurements) and a gas system allowing to flush various gases: Neor Ar-based mixtures with various percentages (between 6 and $20 \%$ ) of $\mathrm{CH}_{4}$ or $\mathrm{CO}_{2}$, or pure $\mathrm{CH}_{4}$ (see Fig. 4). The ionization inside the gas volume has been produced either by $5.9 \mathrm{keV}$ photons from a ${ }^{55} \mathrm{Fe}$ source or $22 \mathrm{keV}$ phonons from ${ }^{109} \mathrm{Cd}$, or by alpha particles $(\sim 5.5 \mathrm{MeV})$ and $60 \mathrm{keV}$ photons from an ${ }^{241} \mathrm{Am}$ source. The signals from the detectors were recorded by standard laboratory electronics-a charge sensitive amplifier Ortec 142PC. In some cases an amplifier Ortec 572 was additionally used. With the help of the scope Le Croy LC 564A we could obtain a pulse height spectrum of the pulses or if necessary convert them to NIM standard pulses and count with a scaler CAEN N145. Pertaining to position resolution measurements (when the ceramic anode plate was used) strips in the central region of the plate were combined in groups (two in each) six of which were individually connected to the Ortec 142PC preamplifiers.

At high gains $\left(>10^{5}\right)$ and also for measurements in the sparking mode a custom made current amplifier was used (developed by J.C. Santuard) in which the feedback resistor and thus the sensitivity can be adjusted to the particular experimental conditions. This allows comparing discharge current values in detectors with metallic electrodes, for example TGEM, with 


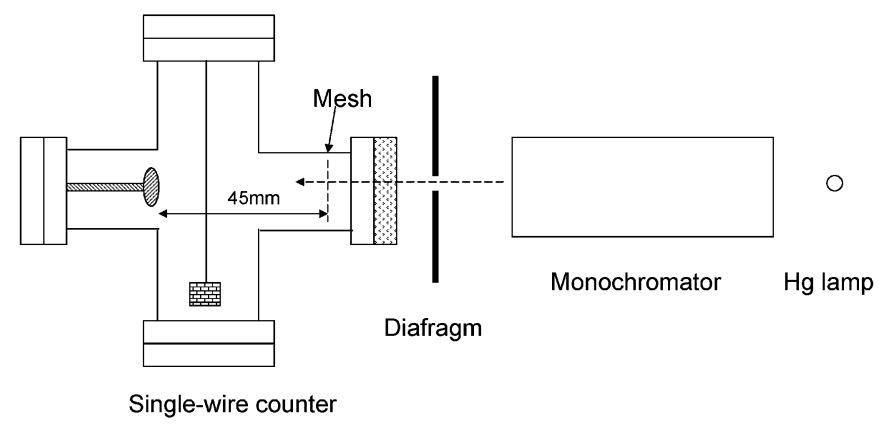

Fig. 5. A schematic drawing of a single-wire counter used for the calibration of the absolute intensity of the UV beam from the monohromator.

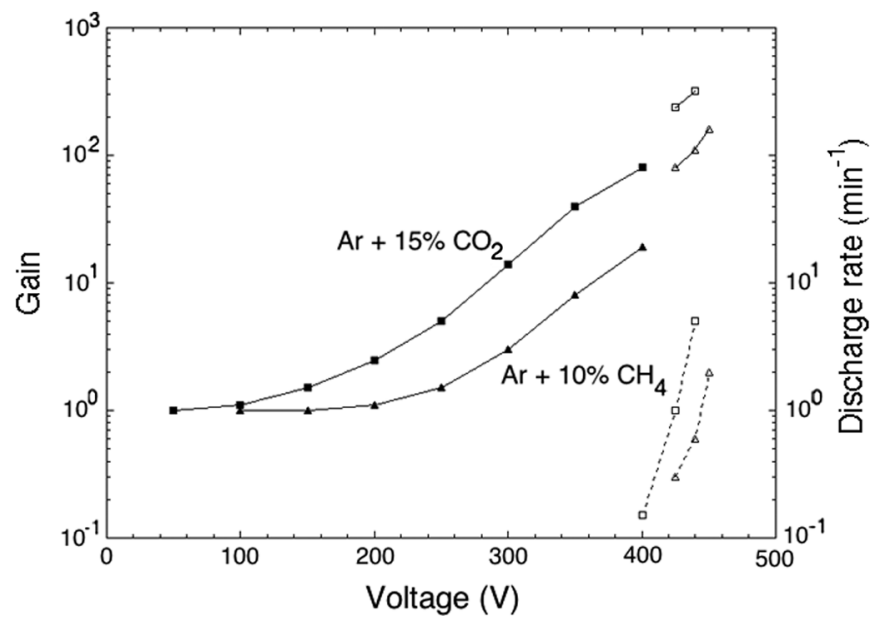

Fig. 6. The gas gain and discharge rate dependence on the anode voltage for the "home-made" $\mathrm{RM}-\mu \mathrm{M}(\mathrm{G}=0.1 \mathrm{~mm}$, fishing line spacers) operating in $\mathrm{Ar}+10 \% \mathrm{CH}_{4}$ (squares) and in $\mathrm{Ar}+15 \% \mathrm{CO}_{2}$ (triangles). Filled symbolsresults obtained with alpha particles, open symbols- $6 \mathrm{keV} \mathrm{X}$-rays. The drift field in these measurements was $150 \mathrm{~V} / \mathrm{cm}$. In the right lower corner of the figure the dash lines represent discharge rates measured with ${ }^{55} \mathrm{Fe}$ in $\mathrm{Ar}+10 \% \mathrm{CH}_{4}$ (squares) and in $\mathrm{Ar}+15 \% \mathrm{CO}_{2}$ (triangles).

those having resistive mesh electrodes. For independent evaluation of the relative energies released by sparks their light intensity we also recorded with a PMT EMI-9426.

For the calibration of the absolute intensity of the light beam exiting the monochromator a single-wire counter was used flushed with a mixture of $\mathrm{CH}_{4}$ with TMAE vapors at temperature of $30^{\circ} \mathrm{C}$ (see Fig. 5). The depth of the active region of this detector was about $4.5 \mathrm{~cm}$ ensuring full UV light absorption inside its volume. In case of measurements in current mode picoammeter Keithely 487 was used.

\section{RESULTS}

\section{A. Bare Resistive Meshes}

Figs. 6-9 show the gain vs. voltage curves measured for various $\mathrm{RM}-\mu \mathrm{M}$ designs having different gap widths. The measurements were stopped at voltages when breakdowns occurred; their rates versus the voltage are also marked in these figures by dash lines. As evident with alpha particles, the maximum achievable gains were around 100 which roughly correspond to the value expected from the Raether limit-see formula (2). However, the maximum gains achieved with ${ }^{55} \mathrm{Fe}$

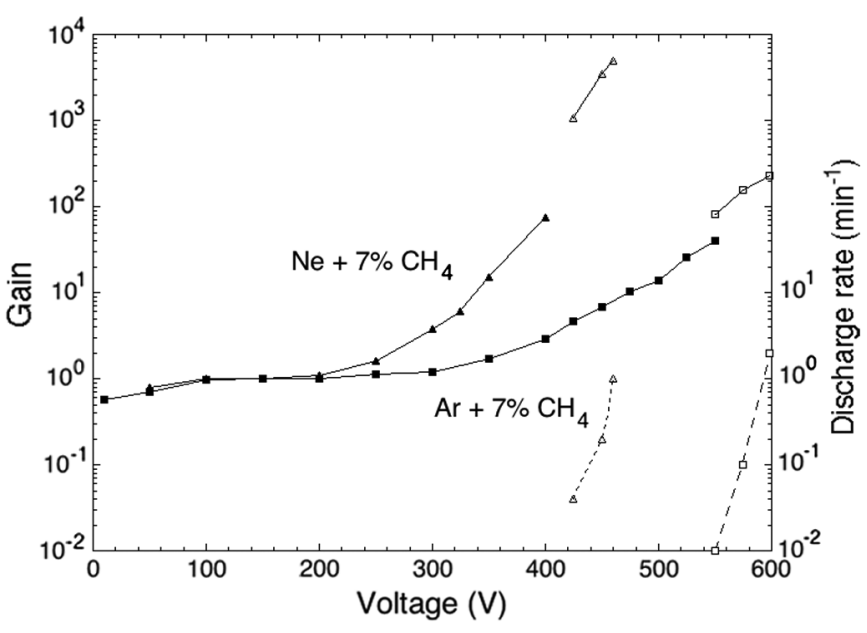

Fig. 7. Gain curves and discharge rate for bulk RM- $\mu \mathrm{M}, \mathrm{G}=0.12 \mathrm{~mm}$, measured in $\mathrm{Ne}+7 \% \mathrm{CH}_{4}$ (triangles) and in $\mathrm{Ar}+7 \% \mathrm{CH}_{4}$ (squares). As in the previous figure the filled symbols represent results obtained with alpha particles, open symbols- $6 \mathrm{keV} \mathrm{X}$-rays and in the right lower corner the dash lines are presenting discharge rates measured with ${ }^{55} \mathrm{Fe}$. Drift filed was $150 \mathrm{~V} / \mathrm{cm}$.

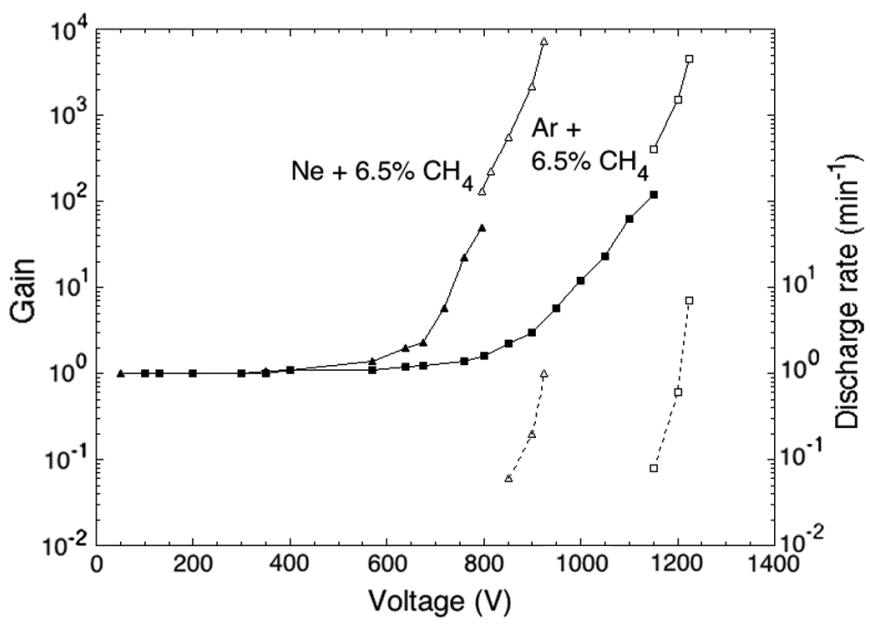

Fig. 8. Gain and discharge rate measurements performed with the "home-made" RM- $\mu \mathrm{M}$, (fishing lines spacers, $\mathrm{G}=0.2 \mathrm{~mm}$, in $\mathrm{Ne}+6.5 \% \mathrm{CH}_{4}$ and in $\mathrm{Ar}+6.5 \% \mathrm{CH}_{4}$. Filled symbols-alpha particles, open symbols-6 keV $\mathrm{X}$-rays from ${ }^{55} \mathrm{Fe}$. The breakdown rate vs. the voltage is indicated with dash lines. The drift field was $150 \mathrm{~V} / \mathrm{cm}$.

(when the voltage on the detector was increased) were much below the calculated one from formula (1). In this case, we think that the discharges were triggered rather by electrodes imperfections than by $6 \mathrm{keV}$ photons. Indeed, without the ${ }^{55} \mathrm{Fe}$ source the voltages at which breakdowns appear were even slightly lower than with the source (indicating also the existence of some minor charging up effect). One of the problems may derive from spacers: gas gains obtained with thin-gap RM-MICROMEGAS $(\mathrm{G}=0.1-0.3 \mathrm{~mm})$ were always rather low (below 10) employing Kapton spacers (these results are not shown) whereas the maximum achievable gains were close to $10^{2}$ (see Fig. 6) using fishing line spacers.

Note that in Ar-based mixtures there was only a slight dependence (a factor 2-3) of the maximum achievable gain on the gas composition $\left(\mathrm{CO}_{2}\right.$ versus $\left.\mathrm{CH}_{4}\right)$ and its percentage (see for example, results depicted in Fig. 6). The same tendency (only 


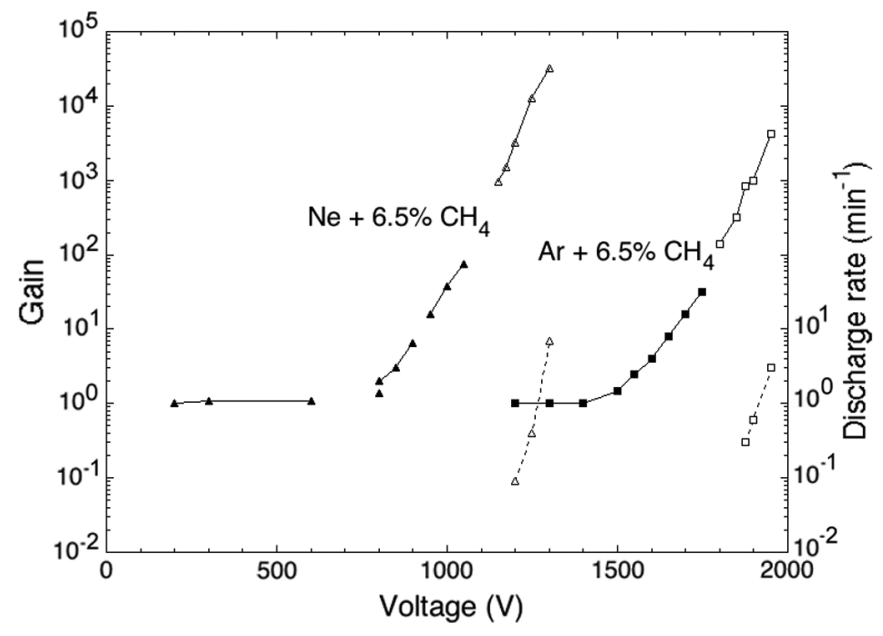

Fig. 9. Gain and discharge rate vs. the voltage for thick-gap RM- $\mu \mathrm{M}(\mathrm{G}=$ $0.5 \mathrm{~mm}$, no spacers). Symbols represent the same measurements as depicted in Fig. 7. To ensure the gap uniformity the active area of this detector was restricted to $1 \times 1 \mathrm{~cm}^{2}$.

TABLE I

SUMMARY OF RESULTS OBTAINED With VARIOUS DESIGNS OF RM- $\mu$ M IN Arand Ne-BAsed Mixtures (See Curves Presented in Figs. 6-9)

\begin{tabular}{|l|l|l|l|}
\hline $\begin{array}{l}\text { Detector } \\
\text { type }\end{array}$ & Gas mixture & $\begin{array}{l}\text { Avalanche } \\
\text { gap (mm) }\end{array}$ & $\begin{array}{l}\text { Maximum } \\
\text { achievable } \\
\text { gain }\end{array}$ \\
\hline RM- $\mu \mathrm{M}$ & Ar-based & 0.1 & $(2-3) \times 10^{2}$ \\
\hline $\begin{array}{l}\text { Bulk RM- } \\
\mu \mathrm{M}\end{array}$ & $\begin{array}{l}\text { Ar-and Ne- } \\
\text { based }\end{array}$ & 0.12 & $\begin{array}{l}2 \times 10^{2} \text { and } \\
5 \times 10^{3}\end{array}$ \\
\hline RM- $\mu \mathrm{M}$ & $\begin{array}{l}\text { Ar -and Ne- } \\
\text { based }\end{array}$ & 0.2 & $\begin{array}{l}4 \times 10^{3} \text { and } \\
7 \times 10^{3}\end{array}$ \\
\hline RM- $\mu \mathrm{M}$ & $\begin{array}{l}\text { Ar -and Ne- } \\
\text { based, no } \\
\text { spacers }\end{array}$ & 0.5 & $\begin{array}{l}5 \times 10^{3} \text { and } \\
3 \times 10^{4}\end{array}$ \\
& & \\
\hline
\end{tabular}

slight dependence on quenchers) was observed in Ne-based mixtures; however in later trials, the maximum achievable gains were always 3-5 times higher than in Ar-based mixtures (see Figs. 7-9).

The results obtained with $\mathrm{RM}-\mu \mathrm{Ms}$ are summarized in Table I. It is observed that the maximum achievable gain monotonically increases with the gap width. As a rule, higher gains were reached with large-gap designs $(\mathrm{G}=0.2-0.5 \mathrm{~mm})$ manufactured without inner spacers between the resistive mesh and the metallic anode plate. Figs. 8 and 9 show gain curves for $\mathrm{RM}-\mu \mathrm{M}$ having $\mathrm{G}=0.2$ and $0.5 \mathrm{~mm}$ respectively: the gains achieved with these detectors pertaining to ${ }^{55} \mathrm{Fe}$ were close to the Raether limit. The energy resolution obtained in $\mathrm{Ar}+\mathrm{CH}_{4}$ with ${ }^{55} \mathrm{Fe}$ radioactive source was about $30-35 \%$ similar as in the example of RETGEM [10].

Fig. 10 depicts the mean signal amplitude vs. the counting rate for RM-MICROMEGAS, $\mathrm{G}=0.2 \mathrm{~mm}$ with metallic anode. The measurements were performed up to the counting rate of $4.7 \mathrm{kH} / \mathrm{cm}^{2}$-the maximum counting rate available from our ${ }^{55} \mathrm{Fe}$ source; no gain drop was observed thus far. As follows from [23] the electrode resistivity can be optimized for higher rates and this will be the subject of the future studies.

The gas gain achieved with RM-GEMs $(\mathrm{G}=0.05-0.01 \mathrm{~mm})$ made from two parallel resistive meshes separated by Kapton

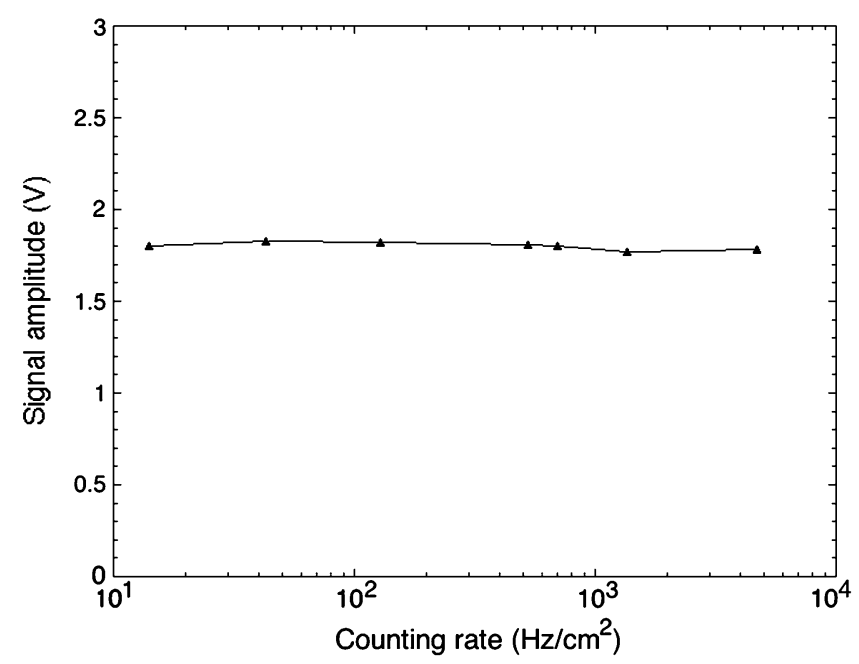

Fig. 10. Counting rate characteristics of RM-MICROMEGAS ( $\mathrm{G}=0.2 \mathrm{~mm}$ ), measured with ${ }^{55} \mathrm{Fe}$ source at a gas gain of 2000 in $\mathrm{Ne}+8 \% \mathrm{CH}_{4}$.

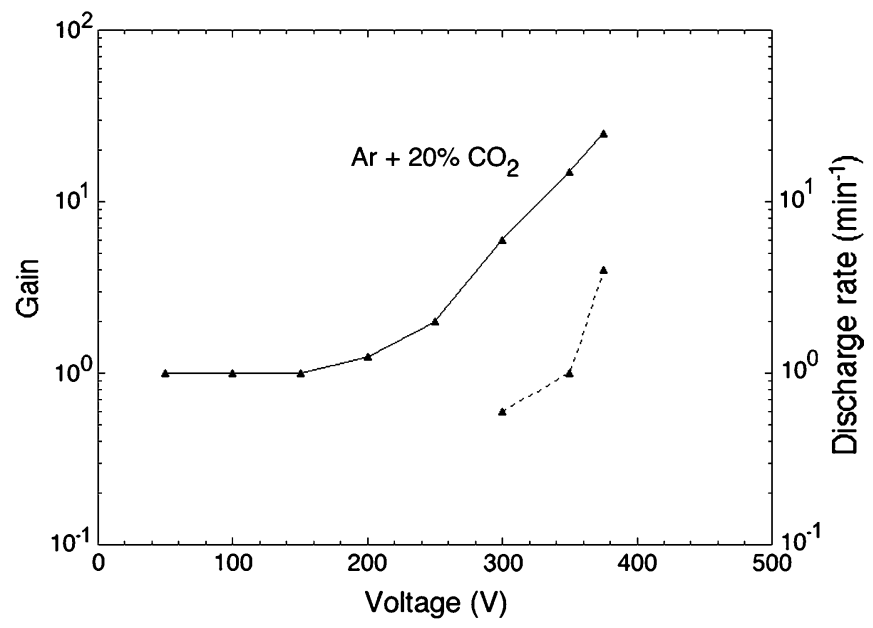

Fig. 11. The gain and discharge rate vs. the voltage for RM-GEM, $\mathrm{G}=0.05 \mathrm{~mm}$ (Kapton spacers) operating in $\mathrm{Ar}+20 \% \mathrm{CO}_{2}$. These measurements were performed with alpha particles. Drift filed $250 \mathrm{~V} / \mathrm{cm}$.

spacers were not high (see Fig. 11), probably due to the Kapton spacers used in these designs and mesh defects. However, RM-MICROMEGAS combined with RM-GEM based preamplifier exhibited an overall gain close to $10^{4}$ (Fig. 12). The measurements presented in this graph were performed only at voltages below some "critical" values at which the rate of discharges reached $\sim 0.1 \mathrm{~min}^{-1}$. However, at higher applied voltages and, thus higher discharge rates, this double or triple step multiplication structure was extensively used to investigate if there is any discharge propagation from one multiplication element to another one. In fact, at any rate of the discharges at any of these amplification elements the discharge propagation was not observed.

One should note that resistive meshes do not provide higher gains or less sparking rate (in good quality detectors both are determine by the Raether limit) compared to the metallic electrodes; their role is to reduce the discharge current and thus ensure less destructive power. The spark current measured during discharges was very different in the case of detector 


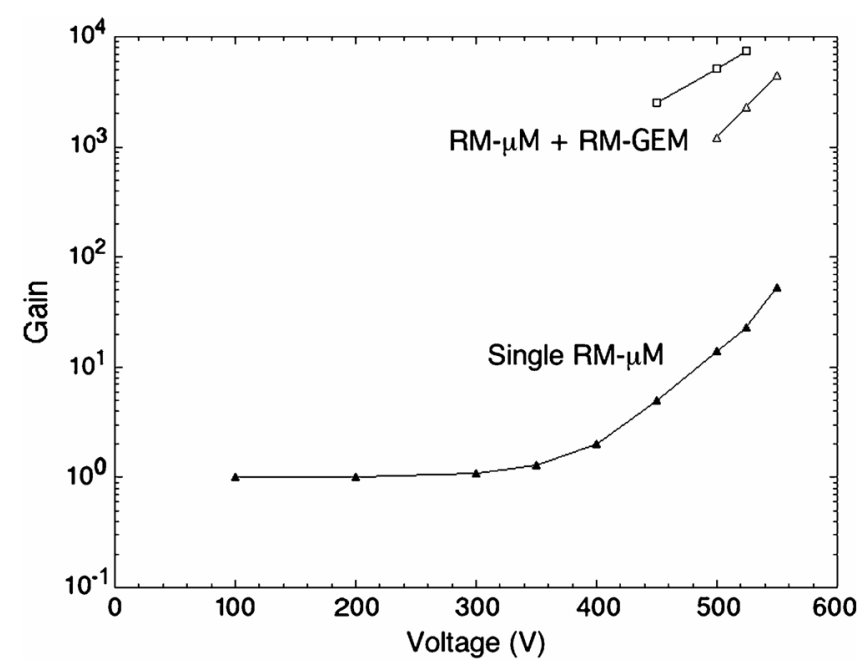

Fig. 12. Results of gain measurements performed with RM-MICROMEGAS, $\mathrm{G}=0.1 \mathrm{~mm}$ using alpha particles (filled rhombus) and with cascaded RM-MICROMEGAS combined with one (open rhombus) or two (open triangles) RM-GEM, G $=0.2 \mathrm{~mm}$ using ${ }^{55} \mathrm{Fe}$ source. Gas mixture $\mathrm{Ar}+15 \% \mathrm{CO}_{2}$; voltage drop over RM-GEM $\Delta \mathrm{V}=700 \mathrm{~V}$ (in the case of one RM-GEM preamplifier), transfer field $1.5 \mathrm{kV} / \mathrm{cm}$. In the case of the double RM-GEM preamplifier the voltage drop over the top RM-GEM was $600 \mathrm{~V}$ whereas on the bottom one $\Delta \mathrm{V}=700 \mathrm{~V}$.

with metallic electrodes and in the case of detectors assembled from the resistive meshes. For example, in the case of Thick GEM (TGEM) with metallic electrodes the discharge current was typically more than $40 \mathrm{~mA}$ whereas in the case of resistive mesh detectors it was not more than 40-200 $\mu \mathrm{A}$ depending on a particular design and the gas used. The light signal produced by sparks in TGEM was at least 10-20 times larger than in resistive mesh detectors.

As was already mentioned above, even at very high transfer electric field between the RM-GEM and RM-MICROMEGAS $(>2 \mathrm{kV} / \mathrm{cm})$ there was no discharge propagation between the detectors. The strong suppression of the discharge energy in the resistive mesh-based detectors is the obvious explanation; consequently, the reduction of the spark's UV emissions which are responsible for the discharge propagation [7].

The main conclusion of these studies indicates that the resistive meshes are convenient construction blocks for various designs of spark-protected detectors.

\section{B. CsI Coated Meshes}

One of the aims of our work was to investigate the possibility of using resistive mesh-based detectors for detection of UV photons with high position resolutions. There are several applications which require such detectors (see for example [5], [11], [24], [25].

As shown in [11], RETGEM-made of resistive Kapton with the cathode coated with a CsI layer-becomes a photosensitive detector able to detect with a high quantum efficiency single UV photons. Unfortunately, as noted in the introduction, the position resolution of the RETGEM is quite modest $(0.7-1 \mathrm{~mm})$ [15], [16] due to the large hole's diameters and pitch.

A CsI coated resistive mesh, having a smaller hole pitch than the RETGEM, allows for the building of thin gap photosensitive detectors with improved position resolutions.

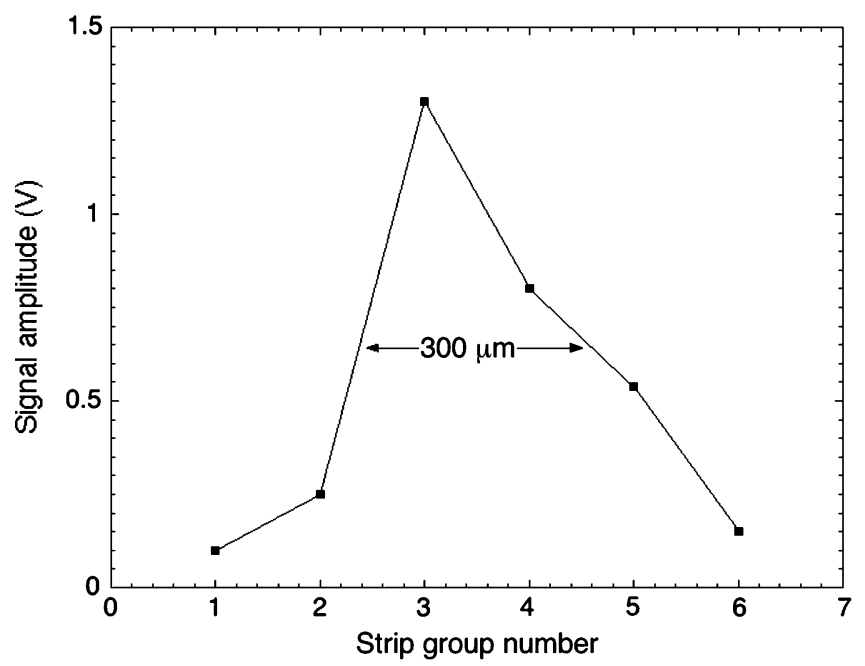

Fig. 13. Signal amplitude vs. the strip group number measured with RM-MICROMEGAS, $\mathrm{G}=0.2 \mathrm{~mm}$ in $\mathrm{Ar}+15 \% \mathrm{CO}_{2}$ at a gas gain of 300 . Drift field $150 \mathrm{~V} / \mathrm{cm}$.

To demonstrate this, we used an CsI coated (the thickness of the CsI layer was $0.4 \mu \mathrm{m}$ ) RM-MICROMEGAS with the ceramic anode plate consisting of metallic readout strips. The light from the pulsed $\mathrm{D}_{2}$ lamp was focused on the top of this mesh (the diameter of the light spot was about $150 \mu \mathrm{m}$ ). The mean value of the pulse amplitude measured versus the strips group number is shown in Fig. 13. As is evident, the width of this distribution at its "half maximum" is about $300 \mu \mathrm{m}, 2-3$ times better than was obtained earlier with a RETGEM.

It was also very important to estimate so-called "photoelectrons collection efficiency": what fraction of the photoelectrons $\mathrm{K}$ emitted from the photocathode (deposited on the top mesh surface) can be drifted through the holes to the inner volume of the detector between the mesh and the anode and collected on the anode plate. This can be rather simply evaluated in the current mode when the detector operates without any multiplication. For this, we measured a photocurrent collected on the drift mesh as a function of the voltage drop between the drift mesh the resistive mesh (in these measurements the resistive mesh was electrically interconnected to the anode plate)-see Fig. 4. Typically, this current increases with the applied voltage and then reaches the plateau value $I_{d}$. We then measured the photocurrent at the anode plate $I_{a}$ as a function of the voltage between the anode and the resistive mesh at the situation where the voltage drop between the drift and the resistive meshes was kept at zero (see a current flow diagram in Fig. 14 in which currents $I_{d}$ and $I_{a}$ are schematically indicated)). For these conditions the collection efficiency can be defined as

$$
\mathrm{K}=\mathrm{I}_{\mathrm{a}} / \mathrm{I}_{\mathrm{d}}
$$

Typical results are depicted in Fig. 15 showing that even at rather small voltages $(100-200 \mathrm{~V})$ at least $80 \%$ of photoelectrons are drifted to the anode-cathode gap. It is clear that the presented above measurements of collection efficiency $\mathrm{K}$ at gain one provides a lower limit of this parameter. For RM- $\mu \mathrm{M}$ and RM-GEMs the results were 70-80\% depending of design and gas mixture. Interesting, to note that with the reversed drift filed 




Fig. 14. The current flow diagram in the case of the measurements of the photoelectrons collection efficiency $\mathrm{K}$.

the collection efficiency slightly increased (about 10\%). Finally, we estimated the quantum efficiency of the CsI layer evaporated on the top of the resistive mesh. For this the top mesh was irradiated by a $185 \mathrm{~nm}$ light from the monochromator and the photocurrent $\mathrm{I}_{\mathrm{a} 1}$ was measured at this wavelength between the resistive mesh and the drift mesh (Fig. 16). Then the same beam from the monochromator was directed to a single-wire counter flushed with the mixture of $\mathrm{CH}_{4}$ and TMAE vapors. The photocurrent created at this point was measured at its saturated/plateau value $\mathrm{I}_{\text {ref }}$. By comparison these photocurrents the quantum efficiency $\mathrm{Q}_{185}$ was estimated:

$$
\mathrm{Q}_{185}=\mathrm{Q}_{\mathrm{TMAE}} \mathrm{I}_{\mathrm{a} 1} / \mathrm{I}_{\mathrm{ref}}
$$

where $\mathrm{Q}_{\mathrm{TMAE}}$ is the TMAE quantum efficiency at $185 \mathrm{~nm}$ $\left(\mathrm{Q}_{\mathrm{TMAE}} \approx 35 \%\right.$-see for example [26]). As depicted in Fig. 16 the ratio $\mathrm{I}_{\mathrm{a} 1} / \mathrm{I}_{\text {ref }}$ is about 0.4 which gives the quantum efficiency value $\mathrm{Q}_{185} \sim 14 \%$ at $185 \mathrm{~nm}$ (note that, due to the holes, the CsI coated surface is about $75 \%$ of the total area, thus the quantum efficiency of the coated area is respectively higher- $\approx 18 \%$ at $185 \mathrm{~nm}$ which is close to one which can be achieved with metallic surfaces). The detection efficiency (D) of the mesh detector at $185 \mathrm{~nm}$ is:

$$
\mathrm{D}=\mathrm{KQ}_{185}
$$

and is at least $11 \%$. It is very probable that at high gains the value of $\mathrm{K}$ reaches $100 \%$ (see [27]) and thus the expected D is around $14 \%$. The quantum efficiency of this mesh was monitored for several months and no degradation was observed so far. Note that the similar results were earlier obtained by us with CsI coated RETGEMS: no degradation of its efficiency was observed during one year. Thus, the preliminary results described above indicate that the resistive mesh may serve as an efficient photocathode for various spark-protected photo detectors.

However, as was already mentioned in the Introduction, the detection of single photoelectrons is not a simple task since in the presence of the radioactive background the maximum achievable gain is determined by events producing high values of primary ionization $\mathrm{n}_{0}$. We are currently considering two ways to overcome this problem:

1) to use designs having high value of the Rather limit, for example cascaded detectors or detectors having wide avalanche gaps (as follows from our earlier studies [2],

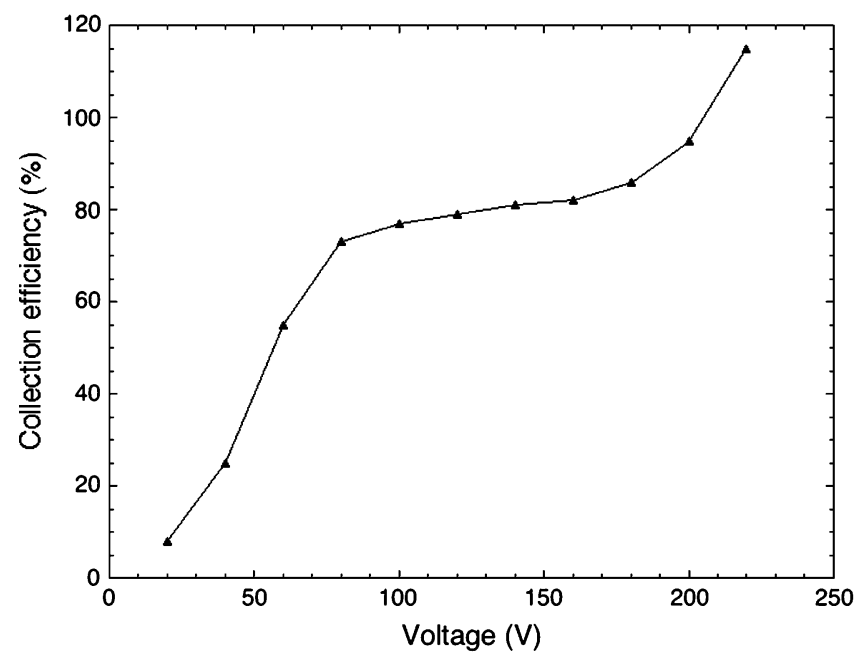

Fig. 15. Photoelectrons collection efficiency K (see formula (3)) vs. the voltage between the resistive cathode mesh and the anode for RM-GEM, G $=0.05 \mathrm{~mm}$ in $\mathrm{CH}_{4}$. Note that at $\mathrm{V}>200 \mathrm{~V}$ the amplification starts, however it is clear that in the voltage interval $100-200 \mathrm{~V}$ the collection efficiency is about $80 \%$.

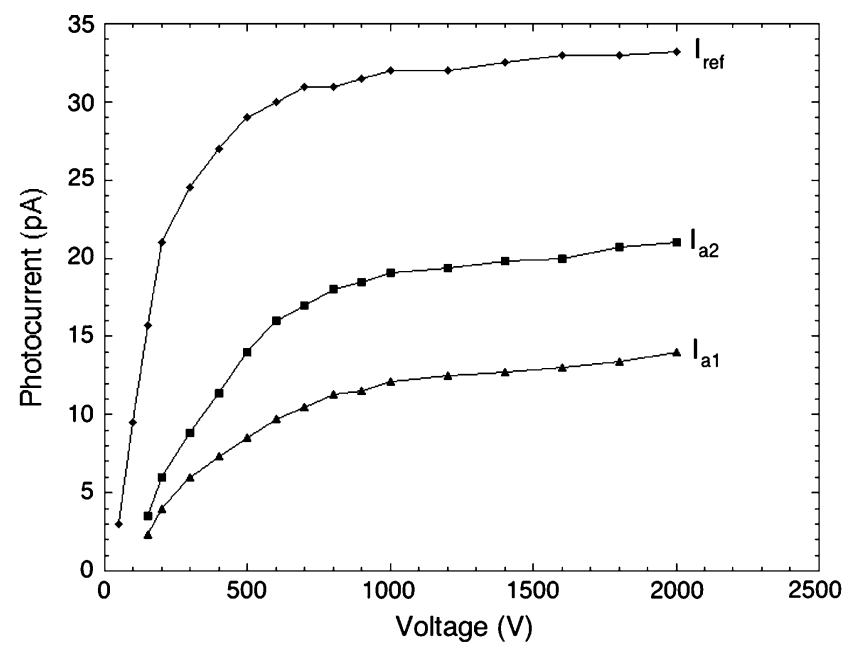

Fig. 16. Photocurrents $\mathrm{I}_{\mathrm{ref}}$ and $\mathrm{I}_{\mathrm{a} 1}$ vs. the applied voltages measured in the single-wire counter $\left(\mathrm{CH}_{4}+\mathrm{TMAE}\right)$ and in between the drift mesh and the resistive cathode meshes $\left(\mathrm{CH}_{4}\right)$ respectively. The current $\mathrm{I}_{\mathrm{a} 2}$ represents the measurements performed in $\mathrm{Ne}+8 \% \mathrm{CH}_{4}$ saturated with ethylferrocene vapors (see Fig. 17)

see also Table I, the maximum achievable gain increases with the gap width $G$ );

2) the use reversed drift field to suppress ionization background as was implemented in [28].

As an illustration of these approaches in Fig. 17 are shown gains versus voltage curves for a $\mathrm{RM}-\mu \mathrm{M}$ having $\mathrm{G}=1 \mathrm{~mm}$ (Nylon spacers), active area $10 \times 10 \mathrm{~cm}^{2}$ and operating in the $\mathrm{Ne}+8 \% \mathrm{CH}_{4}$ saturated with ethylferrocene $(\mathrm{EF})$ vapors. EF vapors were added in order to increase the CsI quantum efficiency (see Fig. 16 and [29], [30]) and at the same time to suppress ion feedback [31]. As can be seen, with UV light gains up to $10^{5}$ were achieved. When the detector was simultaneously irradiated with UV and X-rays from the ${ }^{109} \mathrm{Cd}$ source over the entire sensitive area (the counting rate was $3 \times 10^{5} \mathrm{~Hz}$ ), the maximum achievable gain dropped to $3 \times 10^{3}$ (due to the combination of the Raether limit and the "area effect" [2]), however when the 


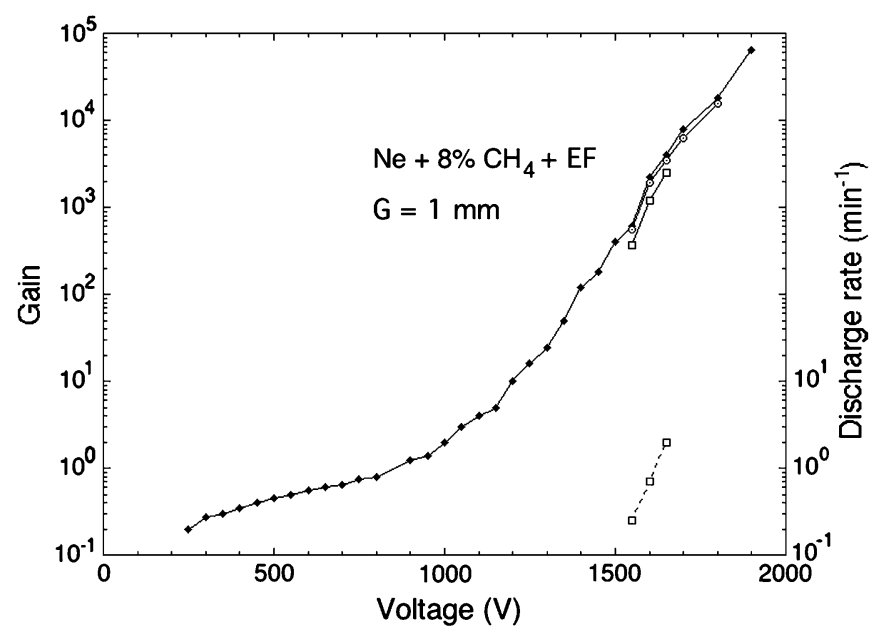

Fig. 17. The gain and the discharge rate vs. the voltage curves for the large gap RM- $\mu \mathrm{M}$ cathode of which was coated with a CsI layer. Filled rhombus-gains measured in current mode with UV light. Open squares-the gain measured in pulsed mode when the detector was simultaneously irradiated with UV light and X-ray photons. The drift field was $200 \mathrm{~V} / \mathrm{cm}$. Dash line with open squaresbreakdown rate measured at these conditions. Open circles represent gain vs. the voltage measured in current mode (UV+X-rays) when the drift field had reversed polarity.

drift filed was reversed (to suppress ionization from the ${ }^{109} \mathrm{Cd}$ ) and its value was $50 \mathrm{~V} / \mathrm{cm}$ a very stable operation at gains up $2 \times 10^{4}$ was achieve in the presence UV+X-rays. With this reversed drift field no discharges were observed at a gain $2 \times 10^{4}$ or below during 7 hours of continuous operation.

In the gas mixture we used in these measurement the quantum efficiency measured at $185 \mathrm{~nm}$ was $\mathrm{Q}_{185}=21 \%$ (see Fig. 16), $\mathrm{K} \approx 80 \%$ (before the multiplication started) and thus the estimated detection efficiency at $185 \mathrm{~nm}$ in the reversed drift filed mode is $\mathrm{D}=18 \%$ (see formula (5)). As it was already mentioned earlier, at high gain operation the collection efficiency most probably is around $100 \%$ so in reality the quantum efficiency of this detector may be as high as $26 \%$.

The results described above are of course preliminary but they are encouraging. Our future studies will be focused on optimization of several resistive mesh-based detector designs (single-stage as well as cascaded) in order to achieve at the same time the highest possible gas gain in the presence of the radioactive background and the best possible position resolution.

\section{CONCLUSIONS}

Resistive meshes developed and tested in this work are convenient construction blocks for various spark-protected detectors including the GEM-like and MICROMEGAS-like.

Due to the holes' small diameter and fine pitch, a better position resolution can be achieved with these RM detectors than with the RETGEMs.

No discharge propagation was observed in our experiment when the RM detectors operated in cascade mode. One of the advantages of the cascade mode is the possibility of reducing an ion back flow to the cathode (see for example [32], [33]). This serves as an attractive feature for some applications such as photodetectors or TPC. Therefore, we believe that RM detectors offer a multitude of applications.
Our future work will be focused on the mesh geometry optimization and on tests of various mesh-based designs in order to achieve higher gas gains and better position resolutions.

\section{ACKNOWLEDGMENT}

Authors would like to thank Ms. M. Louise Nudo for her contribution at the final stage of this work.

\section{REFERENCES}

[1] P. Fonte, V. Peskov, and B. Ramsey, "The fundamental limitations of high-rate gaseous detectors," IEEE Trans. Nucl. Sci, vol. 46, pp. 321-325, 1999.

[2] V. Peskov, P. Fonte, M. Danilesson, and C. Iacobaeus et al., "The study and optimization of new micropattern gaseous detectors for high-rate applications," IEEE Trans. Nucl. Sci, vol. 48, pp. 1070-1074, 2001.

[3] H. Raether, Electron Avalanches and Breakdown in Gases. London, UK: Butterworth, 1964.

[4] P. Martinengo, E. Nappi, R. Oliveira, and V. Peskov et al., "A new generation of GEM detectors and their applications," Nucl. Instrum. and Methods, vol. A604, pp. 8-10, 2009.

[5] J.-M. Bidault, I. Crotty, A. Di Mauro, and P. Martinengo et al., "The first applications of newly developed gaseous detectors with resistive electrodes for UV imaging in daylight conditions," Nucl. Instrum. and Methods, vol. A580, pp. 1036-1041, 2007.

[6] P. Fonte, V. Peskov, and B. Ramsey et al., "A study of breakdown limit in microstrip gas counters with preamplification structures," $\mathrm{Nucl}$. Instrum. and Methods, vol. A416, pp. 23-31, 1998.

[7] C. Iacobaeus, A. Brahme, M. Danielsson, and P. Fonte et al., "A novel portal imaging device for advanced radiation therapy," IEEE Trans. Nucl. Sci., vol. 48, pp. 1496-1502, 2001.

[8] R. Bellazzini, L. Baldini, A. Brez, and F. Cavalca et al., "Gas pixel detectors," Nucl. Instrum. and Methods, vol. A572, pp. 160-167, 2007.

[9] A. Bamberger, K. Desch, U. Renz, and M. Titov et al., "Triple-GEM operated in different gases with highly pixelized readout using MediPix2 chip," Nucl. Instrum and Methods, vol. A572, pp. 157-159, 2007.

[10] R. Oliveira, V. Peskov, F. Pietropaolo, and P. Picchi, "First tests of thick GEMs with electrodes made of a resistive Kapton," Nucl. Instrum. and Methods, vol. A576, pp. 362-366, 2007.

[11] A. Di Mauro, B. Lund-Jensen, P. Martinengo, and E. Nappi et al., "Development of innovative micropattern gaseous detectors with resistive electrodes and first results of their applications," Nucl. Instrum. and Methods, vol. A581, pp. 225-231, 2007, a more detailed version can be found at http://arxiv.org/ftp/arxiv/papers/0706/0706.0102.pdf.

[12] R. Akimoto, H. Hamagaki, T. Gunji, and Y. L. Yamaguchi et al., "Measurement of the basic feature of thick-GEM and resistive-GEM," JINST, vol. 5, pp. 1-8, 2010, P03002.

[13] A. Bondar, A. Buzulutskov, A. Grebenuk, and D. Pavlyuchenko et al. "Thick GEM versus thin GEM in two-phase argon avalanche detectors," JINST, vol. 3, pp. 1-20, 2008, P07001.

[14] V. Razin, B. M. Ovchinnikov, A. I. Reshetin, and S. N. Filippov, RETGEM With Polyvinylchloride (PVC) Electrodes arXiv:0911.4807, pp. 1-5, 2009.

[15] P. Fonte, P. Martinenego, E. Nappi, and R. Oliveira et al., "Progress in developing hybrid RPC: GEM-like detectors with resistive electrodes," Nucl. Instrum. and Methods, vol. A602, pp. 850-853, 2009.

[16] M. Cortesi, V. Peskov, G. Bartesaghi, and J. Miyamoto et al., "THGEM operation in $\mathrm{Ne}$ and $\mathrm{Ne} / \mathrm{CH}_{4}$," JINST, vol. 4, pp. 1-24, 2009, P08001.

[17] H. Van der Graaf, "New developments in gaseous tracking and imaging detectors," Nucl. Instrum. and Methods, vol. A607, pp. 78-80, 2009.

[18] See reports at the, RD51 Collaboration Meeting CERN, December 2009 [Online]. Available: http://indico.cern.ch/conferenceDisplay.py?confId $=72610$

[19] R. Oliveira, V. Peskov, F. Pietropaolo, and P. Picchi, First Tests of Gaseous Detectors Made of a Resistive Mesh, arXiv:1002.1415, pp. $1-15,2010$

[20] I. Giomataris, R. De Oliveira, S. Andriamonje, and S. Aune et al., "Micromegas in a bulk," Nucl. Instrum. and Methods, vol. A560, pp. 405-408, 2006.

[21] C. Iacobaeus, T. Francke, B. Lund-Jensen, and V. Peskov, "An X-ray scanner prototype based on a novel hybrid gaseous detector," Nucl. Instrum. and Methods, vol. A573, pp. 61-64, 2007. 
[22] T. Alexopoulos, A. A. Altintas, M. Alviggi, M. Arik, and S. A. Cetin et al., "Development of large size Micromegas detector for the upgrade of the ATLAS Muon system," Nucl. Instrum. and Methods, vol. A617, pp. 161-165, 2010.

[23] P. Fonte, N. Carolino, L. Costa, and R. Ferreira-Marques et al., "A spark-protected high-rate detector," Nucl. Instrum. and Methods, vol. A431, pp. 154-159, 1999.

[24] R. Bellazzini, G. Spandre, A. Brez, and M. Minuti et al., "Imaging with the invisible light," Nucl. Instrum. and Methods, vol. A581, pp. 246-253, 2007.

[25] J. Melai, A. Breskin, M. Cortesi, and Y. Bilevych et al., "A UV sensitive integrated Micromegas with timepix readout," Nucl. Instrum. and Methods [Online]. Available: http://arxiv.org/PS_cache/arxiv/pdf/ 1003/1003.2083v1.pdf, submitted for publication

[26] T. France and V. Peskov, "Photosensitive gaseous detectors and their applications," Nucl. Instrum. and Methods, vol. A525, pp. 1-5, 2004.

[27] C. D. R. Azevedo, M. Cortesi, A. V. Lyashenko, and A. Breskin et al., "Towards THGEM UV-photon detectors for RICH: On single-photon detection efficiency in $\mathrm{Ne} / \mathrm{CH}_{4}$ and $\mathrm{Ne} / \mathrm{CF}_{4}, " J I N S T$, vol. 5, pp. 1-12, 2010, P01002.
[28] Z. Fraenkel, A. Kozlov, M. Naglis, and I. Ravinovich et al., "A hadron blind detector for the PHENIX experiment at RHIC," Nucl. Instrum. and Methods, vol. A546, pp. 466-480, 2005.

[29] G. Charpak, P. Fonte, V. Peskov, and F. Saul et al., "Investigation of operation of a parallel-plate avalanche chamber with a CsI photocathode under high gain conditions," Nucl. Instrum. and Methods, vol. A307, pp. 63-86, 1991.

[30] G. Charpak, V. Peskov, D. Scigocki, and D. Lemenovski, "Liquid and solid photocathodes and their applications for fast gaseous particle detectors," IEEE Trans. Nucl. Sci, vol. 26, pp. 623-630, 1991.

[31] V. Biteman, S. Guinji, V. Peskov, and H. Sakurai et al., "Position sensitive gaseous photomultipliers," Nucl. Instrum. and Methods, vol. A471, pp. 205-208, 2001.

[32] A. Brahme, M. Danielsson, C. Iacobaeus, and J. Ostling et al., "Evaluation of a GEM and CAT-based detector for radiation therapy beam monitoring," Nucl. Instrum. and Methods, vol. A454, pp. 136-141, 2000.

[33] D. Mörmann, A. Breskin, R. Chechik, and D. Bloch, "Evaluation and reduction of ion back-flow in multi-GEM detectors," Nucl. Instrum. and Methods, vol. A516, pp. 315-326, 2004. 